\section{Medizinisch-ethische Richtlinien zur Sterilisation geistig behinderter Menschen}

\begin{abstract}
Vorwort
Nach Genehmigung durch den Senat der SAMW am 23. November 1999 unterbreiten wir der Ärzteschaft diese neuen Richtlinien zur Vernehmlassung. Eine Subkommission unter der Leitung von Frau Dr. Ursula Steiner-König, Lyss, hat mit Fachleuten aus verschiedenen Gebieten wie Heilpädagogik, Psychiatrie, Sozialarbeit, Genetik, Gynäkologie, Theologie und Jurisprudenz in zahlreichen Sitzungen die 1981 von der SAMW vorgelegten Richtlinien gründlich überarbeitet. Die seit den 80er Jahren gesammelten Erfahrungen haben gezeigt, dass der gesellschaftliche Wandel und neue medizinische Möglichkeiten eine vollkommene Neubearbeitung der Richtlinien nötig machten. Besonders hervorzuheben ist die Forderung nach grösserer Öffnung gegenüber geistig behinderten Mitmenschen, denen mehr Freiheit und Autonomie zugestanden wird. Dies gilt insbesondere für deren Sexualleben, das früher weitgehend verdrängt oder ganz ignoriert wurde. Unsere heutigen Vorschläge, die nach Anhörung zahlreicher direkt Betroffener formuliert wurden, suchen einen für alle Beteiligten optimalen Kompromiss zwischen der Freiheit Behinderter und der Verantwortung und Belastung ihrer Angehörigen und der Gesellschaft.

Wir danken allen, die unter erheblichen Opfern an Zeit die intensiven Diskussionen geführt und die Formulierung des Textes besorgt haben.

Kritik und Änderungsvorschläge erbitten wir bis am 15. Mai 2000 an das Generalsekretariat der SAMW, Petersplatz 13, 4051 Basel; Fax 061/269 9039 oder e-mail: mail@samw.ch.
\end{abstract}

Prof. M. Vallotton, Genf, Präsident der ZEK Prof. W. Hitzig, Zürich, Vizepräsident der ZEK Prof. E. R. Weibel, Bern, Präsident der SAMW

\section{Präambel}

Unter welchen Voraussetzungen die operative Sterilisation geistig behinderter Menschen sich gegenüber den Betroffenen als verantwortbares ärztliches Handeln darstellt, bildet eine ethisch wie rechtlich vielschichtige und teilweise auch kontroverse Frage. Überdies ist die Thematik angesichts menschenverachtender Missbräuche, wie sie das zu Ende gehende/ vergangene Jahrhundert im Zeichen der Eugenik mehrfach erlebt hat, in der öffentlichen Diskussion in hohem Masse emotional besetzt. Die Auseinandersetzung mit ihr verlangt deshalb nach differenzierten Antworten, die sowohl den wohlverstandenen Interessen der betroffenen Person wie auch ihrem Schutz vor Fremdbestimmung Rechnung tragen. Sehr häufig ist allein die Rede von Frauen, die anstehende Problematik betrifft aber ebenso sehr Männer.

Die Richtlinien der SAMW vom 17. November 1981 stellten entscheidend auf den Gesichtspunkt der Urteilsfähigkeit ab. Sie verlangten als unabdingbare Voraussetzung für den Eingriff eine psychiatrische Begutachtung und Beratung, aus der Diagnose und Prognose der geistigen Behinderung mit hinreichender Sicherheit hervorgehen mussten. Ergab sich, dass die betroffene Person urteilsfähig war, so lag der Entscheid allein bei ihr. Allerdings wurde dem Arzt ${ }^{1}$ aufgetragen, darauf zu achten, dass sie ihre Meinung möglichst frei, in Abwesenheit sie beeinflussender Personen äussern konnte, ihr eine ausreichende Bedenkzeit einzuräumen sowie mögliche Vor- und Nachteile des Eingriffs nach den Umständen des einzelnen Falls sorgsam abzuwägen. Stellte sich hingegen heraus, dass die geistig behinderte Person urteilsunfähig war, wurde der Eingriff für nicht zulässig erklärt.

Die Ausschliesslichkeit dieser Regelung erscheint aus mehreren Gründen als nicht mehr in jeder Hinsicht sachgerecht.

- Urteilsfähigkeit ist kein präzise bestimmbarer Begriff. Ob eine geistig behinderte Person Bedeutung und Tragweite einer operativen Sterilisation angemessen einzuschätzen vermag, lässt sich infolgedessen nicht mit naturwissenschaftlicher Objektivität feststellen, sondern nur auf dem Wege bewertender - durch die Subjektivität des Begutachtenden mitgeprägter - Kommunikation interpretativ erschliessen. Das besagt zugleich, dass trotz fachkundiger und sorgfältiger Abklärung das Ergebnis variieren kann.

- Entsprechendes gilt für die Frage, ob die geistig behinderte Person ihren Entscheid letztlich frei oder aber unter dem bestimmenden Einfluss Dritter gefasst hat. Angesichts solcher, aus prinzipiellen Gründen nicht restlos ausräumbarer Unbestimmtheiten musste die ärztliche Praxis nahezu zwangsläufig in den Verdacht geraten, die Richtlinien zu desavouieren, da sich der scheinbar klare Ausschluss der Sterilisation Urteilsunfähiger, den diese normativ statuieren, in der konkreten Umsetzung nicht für jedermann zweifelsfrei verwirklichen lässt.

- Einrichtungen für geistig behinderte Menschen werden heute in der Regel nicht mehr nach Geschlechtern getrennt geführt. Darin, wie auch in der Einstellung vieler Eltern, spiegelt sich ein verändertes gesellschaftliches Verständnis, das geni-

1 In diesen Richtlinien gilt der Einfachheit halber die männliche Bezeichnung für beide Geschlechter. 
tale Sexualität als Möglichkeit eigener Lebensgestaltung auch Personen zuerkennt, die deren Tragweite nicht in jeder Hinsicht zu überblicken vermögen. Von hier aus liegt es nahe, nicht mehr länger auf die Urteilsfähigkeit als einzig massgebliches Kriterium für die Zulässigkeit des Eingriffs abzustellen. Dieser kann ebenso im wohlverstandenen Interesse einer nicht oder nicht voll urteilsfähigen Person mit geistiger Behinderung liegen, vor allem wenn er darauf gerichtet ist, die Gefahr von Beeinträchtigungen der körperlichen oder geistigen Gesundheit infolge sexueller Kontakte zu vermeiden und wenn dieser Gefahr sonst nur durch freiheitsbeschränkende Massnahmen begegnet werden könnte.

- Verändert hat sich seit dem Erlass der Richtlinien auch die Rechtslage. Während damals keine gesetzlichen Regelungen bestanden, sind mittlerweile kantonale Gesundheitsgesetze in Kraft getreten, welche die Möglichkeit der Sterilisation urteilsunfähiger Personen ausdrücklich vorsehen (s. unten, II 1.2).

Aus diesen Gründen erklären die vorliegenden Richtlinien die Sterilisation Urteilsunfähiger nicht mehr für schlechthin unzulässig. Sie binden sie jedoch an eine Reihe von Voraussetzungen inhaltlicher wie formaler Art, die sicherstellen sollen, dass ein Eingriff erst nach eingehender Abklärung der Umstände des Einzelfalls und allein im überwiegenden Interesse der oder des Betroffenen erfolgt. Diese Voraussetzungen sind ebenso bei geistig behinderten Personen zu beachten, die für urteilsfähig befunden werden. Wegen ihrer generell grösseren Beeinflussbarkeit durch Dritte als Folge ihrer Abhängigkeit sowie der Schwierigkeiten, den Zustand der Urteilsfähigkeit trennscharf von dem ihres Fehlens abzugrenzen, bedürfen auch sie eines besonderen Schutzes vor Fremdbestimmung.

Verlässliche Angaben über die Häufigkeit von Sterilisationen geistig behinderter Menschen in der Schweiz sowie die Gründe, aus denen diese vorgenommen werden, bestehen bis heute nicht. Infolgedessen kursieren in der öffentlichen Diskussion Mutmassungen über "Dunkelziffern", die eine sachliche Auseinandersetzung mit der Thematik erschweren. Um die erwünschte Transparenz zu schaffen, sehen die Richtlinien deshalb die Errichtung eines zentralen, unter Wahrung der Anonymität der Betroffenen zu führenden Registers vor.

\section{Richtlinien}

\section{Rechtliche Aspekte}

\subsection{Bundesrecht}

Das Bundesrecht kennt keine gesetzliche Vorschrift, die sich speziell auf die Sterilisation bezieht. Der Eingriff erfüllt jedoch den Tatbestand der schweren Körperverletzung (Art. 122 StGB) und bedarf deshalb, soll er zulässig sein, der Rechtfertigung durch die Einwilligung der verletzten Person. Ist diese urteilsunfähig, so kommt - wie beim Schwangerschaftsabbruch, wo das Strafgesetz diese Befugnis ausdrücklich vorsieht (vgl. Art. 120 Ziff. 1 Abs. 3 StGB) - eine Einwilligung durch den gesetzlichen Vertreter in Betracht, sofern der Eingriff im überwiegenden Interesse der betroffenen Person liegt.

\subsection{Kantonales Recht}

Besondere Bestimmungen über die Sterilisation enthalten, als bislang einzige Erlasse des kantonalen Rechts, die Gesundheitsgesetze von Aargau und Neuenburg. Nach der Aargauer Regelung ist der Eingriff bei unmündigen und entmündigten Personen zulässig, wenn zwei fachärztliche Gutachten, darunter ein psychiatrisches, ihn befürworten sowie die schriftliche Zustimmung des gesetzlichen Vertreters sowie, falls die betroffene Person urteilsfähig ist, auch deren Einwilligung vorliegen (§ 51 Abs. 2 des Gesundheitsgesetzes von 10.11.1987). Das neuenburgische Recht verlangt für die Sterilisation Unmündiger und Entmündigter stets die schriftliche Zustimmung des gesetzlichen Vertreters und, falls die betroffene Person urteilsfähig ist, zusätzlich deren schriftliche Einwilligung. Bei minderjährigen und urteilsunfähigen Personen ist überdies eine Bewilligung des Kantonsarztes erforderlich, der vorgängig die Stellungnahme eines neutralen Sachverständigen einzuholen hat (Art. 32 des Gesundheitsgesetzes vom 6.2.1995).

\subsection{Europäisches Übereinkommen über Menschen- rechte und Biomedizin}

Nach Artikel 6 Ziffer 1 des Europäischen Übereinkommens über Menschenrechte und Biomedizin vom 4. 4.1997, das demnächst durch die Schweiz ratifiziert werden soll, dürfen medizinische Eingriffe an einwilligungsunfähigen Personen nur vorgenommen werden, wenn sie zu deren «unmittelbaren Nutzen erfolgen». Der Vernehmlassungsbericht des Bundesrates misst diesem Erfordernis indessen nur die Bedeutung $\mathrm{zu}$, die Berücksichtigung von Drittinteressen auszuschliessen, und hält demgemäss die Sterilisation Urteilsunfähiger zur Vermeidung künftiger Beeinträchtigungen der körperlichen oder geistigen Gesundheit durch Schwangerschaft, Geburt oder die spätere Trennung vom Kind als mit dem Übereinkommen für vereinbar.

Die zunehmende Verrechtlichung der Problematik zeigt allerdings, dass das Bedürfnis nach ärztlichen Standesregeln vor allem dort besteht, wo klare rechtliche Vorgaben fehlen. Die Akademie würde deshalb eine gesetzliche Regelung im Rahmen der geplanten Revision des Vormundschaftsrechts begrüssen.

Jeder Mensch muss für sich entscheiden, was Behinderung und Einschränkung für ihn bedeuten und wie er damit umgehen kann. Dies ist keine Frage allein medizinischer Berufsethik; über sie sollte vielmehr in einem demokratisch legitimierten Willensbildungsprozess entschieden werden. Die Richtlinien wollen und können diesen nicht ersetzen. Sie versuchen, den angesichts einer menschlich wie juristisch schwierigen Situation vielfach bestehenden Unsi- 
cherheiten - bei der Ärzteschaft, den betroffenen Eltern und den Betreuenden - soweit zu begegnen, wie es als möglich erscheint.

\section{Standortbestimmung}

\subsection{Beratung geistig behinderter Personen}

Viele geistig behinderte Personen sind urteilsfähig im Hinblick auf schwangerschaftsverhütende Massnahmen, aber auch im Hinblick auf eine allfällige Familiengründung. In individuell angepasster Weise soll ihnen eine umfassende ärztliche und psychologische Beratung unter Einbezug erbbiologischer Zusammenhänge zuteil werden.

Die geistige Behinderung kann aber auch eine verminderte oder fehlende Urteilsfähigkeit implizieren. In diesen Fällen hat die fachärztlich zu beantwortende Frage, ob eine Verbesserung der geistigen Leistungsfähigkeit mit der Erlangung einer Urteilsfähigkeit mit Sicherheit auszuschliessen ist, eine grosse Bedeutung.

Auch bei behinderten Menschen, die bezüglich einer Familiengründung urteilsunfähig sind, schliesst ihr erfülltes Leben Sexualität ein. Sie können auch den Wunsch nach Kindern haben. Ob die Realisierung eines solchen Kinderwunsches möglich ist, hängt u.a. auch von den konkreten Möglichkeiten einer gemeinsamen Betreuung von geistig behinderten Eltern und ihren Kindern ab. In einer genauen Prüfung des Einzelfalles geht es darum, die geistig behinderten Menschen allenfalls auch vor Schaden durch eine Elternschaft zu bewahren.

Der professionell durchgeführten sexualpädagogischen Aufklärung, wie sie sich heute allerdings erst an wenigen Institutionen im Aufbau befindet, wird ein hoher präventiver Stellenwert beigemessen. Solche Aktivitäten sind zu unterstützen.

\subsection{Kontrazeptive Möglichkeiten}

Es steht heute eine Reihe reversibler kontrazeptiver Verfahren mit hoher Sicherheit zur Verfügung, für deren Evaluation im Einzelfall eine eingehende fachärztliche Beratung geboten ist. Psychologischen und heilpädagogischen Aspekten muss unter Beizug von Fachkräften Rechnung getragen werden. Nicht selten erweist sich im probemässigen Einsatz ein reversibles Verfahren entgegen aller Erwartungen als praktikabel.

Die operative Sterilisation, d.h. die Blockierung der Eileiter bei der Frau bzw. der Samenleiter beim Mann, ist streng von der Kastration, die die Entfernung der Keimdrüsen (Eierstöcke bzw. Hoden) bedeutet, zu unterscheiden. Die Kastration und medikamentöse Verfahren mit analoger Zielsetzung, die zu tiefgreifenden Veränderungen der Persönlichkeit führen, stehen hier nicht zur Diskussion.

\section{Ethische Erwägungen}

Das Wohl des geistig behinderten Menschen ist prioritär. Alle Bestrebungen müssen zum Ziele haben, ihm in Entfaltung seiner geistigen und körperlichen Möglichkeiten zu einem glücklichen und erfüllten Leben zu verhelfen. Die Wahrung der Privatsphäre der betroffenen Person und ihrer Familie muss mit der strikten Einhaltung des Berufsgeheimnisses garantiert sein. Grundsätzlich bleibt vor der Diskussion um eine kontrazeptive Massnahme, sei sie reversibel oder im Sinne der operativen Sterilisation, abzuklären, ob bei der von der betroffenen Person praktizierten Sexualität eine Schwangerschaft überhaupt zustande kommen könnte.

\subsection{Interessen von Drittpersonen}

Bei der ärztlichen Beratung und Hilfeleistung zur Durchführung kontrazeptiver Massnahmen, insbesondere einer Sterilisation bei einer geistig behinderten Person, ist zu bedenken, dass dieses Begehren immer von dritter Seite gestellt wird. Die Interessen dieser Dritten, ihre Beziehung zum betroffenen Menschen und die unumgängliche Intersubjektivität in der sich anbahnenden Dreieckskonstellation, die bei jeder psychosozialen Fragestellung weit bedeutsamer zutage tritt als bei somatischen Abklärungen, müssen mit grosser Sorgfalt berücksichtigt und reflektiert werden. Die voraussichtliche Lebensqualität sollte ausschliesslich aus der Perspektive des geistig behinderten Menschen und nicht aus der Sicht der Angehörigen oder des betreuenden Teams beurteilt werden.

\subsection{Sterilisation und sexuelle Gewalt}

Eine Empfängnisverhütung irgendwelcher Art, also auch der Sterilisation, stellt keinen Schutz vor sexueller Gewalt bzw. Gewaltanwendung dar. Ob eine Sterilisation das Risiko einer sexuellen Ausbeutung erhöht, wird kontrovers diskutiert.

Aus ethischen Gründen ist es nicht zulässig, das Risiko einer vergewaltigungsbedingten Schwangerschaft als Begründung für eine Sterilisation beizuziehen. Im selben Sinne stellt das Risiko, dass geistig behinderte Männer sich einer Vergewaltigung schuldig machen könnten, keine Begründung für eine Sterilisation dar.

\subsection{Operative Sterilisation als ultima ratio}

Die operative Sterilisation darf nur als ultima ratio in Betracht gezogen werden. Wenn nach einfühlsamer und den intellektuellen Fähigkeiten der behinderten Person entsprechender Aufklärung eine dauerhafte ablehnende Willensäusserung erkennbar wird, darf der Eingriff nicht durchgeführt werden. Falls Aussicht auf eine Besserung der kognitiven Fähigkeiten besteht, muss der Eingriff ebenfalls unterbleiben. Je jünger die betroffene Person ist, desto zurückhaltender ist der Eingriff in Erwägung zu ziehen.

\section{Praktisches Vorgehen}

\subsection{Erste Massnahmen}

Die ärztliche Aufgabe geht dahin, besorgt zu sein, dass der betroffenen Person eine ihren Verständigungsmöglichkeiten entsprechende Aufklärung zuteil wird und allenfalls schwangerschaftsverhütende Massnahmen getroffen werden. Reversible kontra- 
zeptive Verfahren müssen in jedem Falle als Massnahmen der ersten Wahl betrachtet werden. Sollte trotz allem Bemühen eine reversible Kontrazeption nicht durchführbar sein und bestehen berechtigte Hinweise auf eine sexuelle Aktivität, kann eine operative Sterilisation erwogen werden.

\subsection{Voraussetzungen für eine operative Sterilisation} sind:

- ein unabhängiges psychiatrisches Gutachten;

- eine zweite schriftliche Stellungnahme, die von einer nichtärztlichen, in der Betreuung der betroffenen Person eingebundenen oder auch von einer aussenstehenden Fachperson, die mit den spezifischen Problemen geistig behinderter Menschen vertraut ist, abzugeben ist;

- die schriftliche Zustimmung des gesetzlichen Vertreters.

\section{Variante A}

Das psychiatrische Gutachten und die zweite schriftliche Stellungnahme sind bei der von der Zentralen Ethikkommission (ZEK) der SAMW eingerichteten Koordinationsstelle, Petersplatz 13, CH-4051 Basel, anonymisiert einzureichen.

\section{Variante $B$}

Das psychiatrische Gutachten und die zweite schriftliche Stellungnahme sind bei der gesundheitspolitischen Behörde (Sanitätsdirektion, Gesundheitsdepartement, kantonsärztlicher Dienst) des Wohnkantons der betroffenen Person zu deponieren.

Bei Unklarheiten oder Konflikten bietet die Zentrale Ethikkommission ihre guten Dienste an.

\section{Kommentar}

\section{ad II 1. Rechtliche Aspekte}

\section{ad II 1.2 Kantonales Recht}

In einem dritten Kanton, Freiburg, soll nach dem Vorentwurf des Gesundheitsgesetzes vom Oktober 1997 die Sterilisation urteilsunfähiger Personen zulässig sein, wenn die schriftliche Einwilligung des gesetzlichen Vertreters vorliegt und eine spezielle Kommission (commission de surveillance des professions de la santé et des droits des patients) der Vornahme des Eingriffs mit Zweidrittelsmehrheit zustimmt (Art. $93 \mathrm{VE})$.

Im Gesundheitsgesetz des Kantons Tessin wird auf die Richtlinien der SAMW verwiesen.

\section{ad II 1.3 Europäisches Übereinkommen über Menschenrechte und Biomedizin}

Als Auslegungshilfe für eine mit dem Übereinkommen konforme Anwendung der unter II 1.2 genannten kantonalen Gesundheitsgesetze verweist der Bundesrat auf die detaillierte Regelung durch das deutsche Recht in § 1905 des Bürgerlichen Gesetzbuches (BGB):
(1) Besteht der ärztliche Eingriff in einer Sterilisation des Betreuten, in die dieser nicht einwilligen kann, so kann der Betreuer nur einwilligen, wenn

1. die Sterilisation dem Willen des Betreuten nicht widerspricht;

2. der Betreute auf Dauer einwilligungsunfähig bleiben wird;

3. anzunehmen ist, dass es ohne die Sterilisation zu einer Schwangerschaft kommen würde;

4. infolge dieser Schwangerschaft eine Gefahr für das Leben oder die Gefahr einer schwerwiegenden Beeinträchtigung des körperlichen oder seelischen Gesundheitszustandes der Schwangeren zu erwarten wäre, die nicht auf zumutbare Weise abgewendet werden könnte, und

5. die Schwangerschaft nicht durch andere zumutbare Mittel verhindert werden kann. Als schwerwiegende Gefahr für den Gesundheitszustand der Schwangeren gilt auch die Gefahr eines schweren nachhaltigen Leidens, das ihr drohen würde, weil vormundschaftsgerichtliche Massnahmen, die mit der Trennung vom Kind verbunden wären (§§ 1666, 1666a), gegen sie ergriffen werden müssten.

(2) Die Einwilligung bedarf der Genehmigung des Vormundschaftsgerichts. Die Sterilisation darf erst zwei Wochen nach Wirksamkeit der Genehmigung durchgeführt werden. Bei der Sterilisation ist der Methode der Vorzug zu geben, die eine Refertilisierung zulässt.

\section{ad II 2. Standortbestimmung}

\section{ad II 2.1 Beratung geistig behinderter Personen}

Die Beratung im Hinblick auf schwangerschaftsverhütende Massnahmen hat einen hohen Stellenwert. Vor- und Nachteile der einzelnen Verfahren müssen leicht verständlich erläutert werden.

Erbbiologische Zusammenhänge sind aufzuzeigen. In Fällen ohne sichere Diagnose ist eine genetische Untersuchung zu erwägen oder zu wiederholen, da sich angesichts der raschen Fortschritte der Genetik neue Gesichtspunkte ergeben könnten. Auf eine allfällige genetische Prädisposition mit einem Wiederholungsrisiko für die Nachkommenschaft soll einfühlsam aufmerksam gemacht werden.

\section{ad II 2.2 Kontrazeptive Möglichkeiten}

Die Anwendung reversibler Verfahren hängt vom Grad der geistigen Behinderung der betreffenden Person ab. Von seiten der Ärzte und Betreuer ist für eine eingehende Instruktion zu sorgen. Für die Frau sind in erster Linie die hormonale Kontrazeption (kombinierte orale Kontrazeptiva, orale Gestagenpräparate, Depotgestagene zur Injektion, subdermale Implantate, Vaginalringe) und die Intrauterinpessare (Kupfer-IUP, Gestagen-IUP, inerte Kunststoff-IUP) zu erwähnen. Barrieremethoden (Kondom für die Frau, Diaphragma, Portiokappen, Vaginalschaum, Spermizide) haben eine geringere kontrazeptive Sicherheit, 
und zudem sind ihrer Anwendung bei körperlichen und geistigen Behinderungen rasch Grenzen gesetzt. Das gleiche gilt für natürliche Verfahren und die postkoitale Interzeption.

Die Einstufung des Schwangerschaftsabbruches als kontrazeptive Massnahme ist nicht akzeptabel. Geistig behinderte Frauen sind in engagierter und sorgfältiger Weise vor unerwünschter Schwangerschaft zu schützen.

Für den Mann kommt die Anwendung des Kondoms in Betracht.

Wird die operative Sterilisation erwogen, müssen bei der Beratung der grosse Aufwand und die unsichere Erfolgschance, die Unterbindung rückgängig $\mathrm{zu}$ machen, gebührend Berücksichtigung finden. Zwischen den Beratungsgesprächen und der Operation soll eine angemessene Bedenkzeit eingeschaltet werden. Von der betroffenen Person muss, falls sie urteilsfähig ist, eine unterschriftliche Bestätigung verlangt werden. Ärztlicherseits sind das operative Vorgehen und allfällige Risiken leicht verständlich darzulegen. Diese Erfordernisse müssen einwandfrei dokumentiert und gegenseitig unterzeichnet sein.

Falls die vorgebrachte Argumentation den Arzt nicht überzeugt, steht ihm das Recht zu, den Eingriff abzulehnen. Eine ablehnende Haltung kann er auch aus Gewissensgründen geltend machen.

\section{ad II 3. Ethische Erwägungen}

\section{ad II 3.1 Interessen von Drittpersonen}

Angesichts der äusserst intimen Befragungs- und Informationsaufgabe einerseits und der Mediation zwischen ureigensten Wünschen eines Menschen und den zu respektierenden gesellschaftlichen Regeln andererseits sieht sich der Arzt in besonders betonter Weise in der Rolle der Vertrauensperson. Diese Aufgabe erfordert viel Zeit und grosse Geduld. Es sind verbale und nonverbale Kommunikationsmodalitäten nötig, damit die geistig behinderte Person verstehen kann, dass man ihr nichts wegnehmen, sondern ihr dazu verhelfen möchte, trotz ihrer Behinderung ein möglichst autonomes und kontrollierende Obhut vermeidendes Leben führen zu können, wo intime Kontakte mit der offensichtlichen Möglichkeit einer Schwangerschaft einhergehen.

In Fällen einer stabilen Beziehung wird der Einbezug des Partners oder der Partnerin besonders wichtig sein.

Die Eltern eines geistig behinderten Menschen können die Tatsache verkennen, dass dieses Individuum im Erwachsenenalter nicht einfach als «ewiges Kind" betrachtet werden darf. Dem Arzt erwächst daraus allenfalls die Aufgabe, die veränderte Konstellation und den Umgang damit aufzuzeigen.

Geistig behinderte Eltern könnten durch die Betreuung eines Kindes überfordert sein, und das Kind würde höchstwahrscheinlich eine Fremdplazierung erfahren. Darüber sind Eltern bzw. Grosseltern sehr oft besorgt. Die ärztliche Beratung muss auf diese Sorgen eingehen. Auf die rechtlichen Aspekte des "unmittelbaren Nutzens» für die betroffene Person ist aufmerksam zu machen. Ärztlicherseits sollte auch angeboten werden, die Betroffenen in ihrem Entscheidungsprozess zu begleiten. Je nach Komplexität der individuellen Situation kann eine erweiterte psychiatrische oder psychologische Beratung sinnvoll oder sogar notwendig sein.

\section{ad II 3.2 Sterilisation und sexuelle Gewalt}

Auch die Sorge um eine allfällige Vaterschaftsklage gegen einen geistig behinderten Mann kann nicht als Grund für eine Sterilisation gelten.

\section{ad II 3.3 Operative Sterilisation als ultima ratio}

Bei der Fragestellung um die operative Sterilisation sollen die Eltern der betroffenen urteilsunfähigen Person beratend einbezogen werden, und zwar auch nach dem gesetzlich vorgegebenen Zeitpunkt des Erlöschens der elterlichen Gewalt. Der Mündigkeit kann keine entscheidende Relevanz beigemessen werden. Bei Fehlen der Eltern sind nach sorgfältiger Überprüfung des sozialen Umfeldes allenfalls nächste Angehörige, die an Eltern Statt Sorgeverpflichtungen wahrgenommen haben, anzuhören.

\section{ad II 4. Praktisches Vorgehen}

\section{ad II 4.1 Erste Massnahmen}

Erwachende sexuelle Regungen geistig behinderter Jugendlicher bewegen deren Betreuer (Eltern, gesetzliche Vertreter, Pflegeverantwortliche in Institutionen), die Hilfe und Beratung einer Fachperson ihres Vertrauens in Anspruch zu nehmen. Bei Unsicherheit in der Fragestellung der Kontrazeption soll die betroffene Person für diese spezifische Beratung und Instruktion an eine Familienplanungsstelle überwiesen werden. Vordringlich sind die Erörterung der korrekten Durchführung der gewählten kontrazeptiven Methode und deren Überwachung.

\section{ad II 4.2 Voraussetzungen für eine operative} Sterilisation

Die psychiatrische Begutachtung muss durch einen unabhängigen, d.h. nicht in der Betreuung der betroffenen urteilsunfähigen Person involvierten Facharzt erfolgen. Die zweite zustimmende schriftliche Stellungnahme wird von einer nichtärztlichen Fachperson verlangt, die entweder fest in die Betreuung der betroffenen Person eingebunden ist oder durch ihre eigene praktische Tätigkeit die im individuellen Fall anstehende Problematik in profunder Weise kennt. Die Verteidigung der Rechte geistig behinderter Menschen ist in engagierter Weise wahrzunehmen. Das psychiatrische Gutachten und die erwähnte zweite Stellungnahme müssen sicherstellen, dass der Eingriff im wohlverstandenen und überwiegenden Interesse der betroffenen Person erfolgt. Das ärztliche Gespräch bzw. die Exploration hat teilweise unter Ausschluss der Betreuenden zu erfolgen. Zur Urteilsfähigkeit muss in klarer Weise Stellung bezogen werden.

Die Brisanz der öffentlichen Auseinandersetzung mit der Problematik der operativen Sterilisation geistig behinderter urteilsunfähiger Menschen erfordert 
den Schutz sowohl der betroffenen Personen als auch deren Angehörigen und Betreuer. Aus diesem Grunde werden zwei schriftliche Stellungnahmen, eine davon im Sinne eines psychiatrischen Gutachtens, die

- Variante A: bei der dazu eingerichteten Koordinationsstelle der Zentralen Ethikkommission (ZEK) der SAMW, Petersplatz 13, CH-4051 Basel;

- Variante B: bei der gesundheitspolitischen Behörde des Wohnkantons der betroffenen Person zu deponieren sind, verlangt. Das Ziel liegt in einer breiten und sicheren Abstützung der Verantwortung für das Wohl geistig behinderter Mitmenschen. Die Weiterleitung der anonymisierten beiden Stellungnahmen an eine Koordinationsstelle unter Aufsicht der Zentralen Ethikkommission (ZEK) der SAMW lässt neben der weiteren Absicherung der Verantwortung statistische Erhebungen auf gesamtschweizerischer Ebene mit der damit von weiten Bevölkerungskreisen geforderten Transparenz zu.

[Bemerkung Subkommission: Diese Transparenz wäre nicht gegeben bei alleiniger Meldung an die Gesundheitsbehörden, denn diese würden die Zahlen allenfalls nicht herausgeben - vergl. Schwangerschaftsabbruch!]

Genehmigt von der Zentralen Ethikkommission der SAMW am 22. Oktober 1999

Prof. M. Vallotton, Genf, Präsident der ZEK

Prof. W. Hitzig, Zürich, Vizepräsident der ZEK

Genehmigt vom Senat der SAMW

am 23. November 1999

Prof. E. R. Weibel, Bern, Präsident der SAMW

Mitglieder der für die Ausarbeitung dieser Richtlinien tätigen Subkommission:

Dr. Ursula Steiner-König, Lyss, Präsidentin; Dr. U. Aebi, Bern; Heidi Blaser, Bern; PD Dr. A. Bondolfi, Zürich; Dr. R. Bonfranchi, Bern; Regina EugsterGrossenbacher, Zürich; Prof. W. Felder, Bern; Dr. Monica Gersbach-Forrer, Genf; Prof. Dr. iur. G. Jenny, Bern; Dr. Cornelia Klauser-Reucker, Agno; Prof. W. Stoll, Aarau.

\section{Literatur}

Eser A, Hirsch HA (Hrsg.). Sterilisation und Schwangerschaftsabbruch. "Medizin und Recht». Band 10. Stuttgart: Enke Verlag; 1980.

Shaw J. Sterilisation of mentally handicapped people: judges rule OK? The Modern Law Review 1990;53:91-106.

Finger P. Die Sterilisation geistig Behinderter nach Art. 1905 BGB in der Fassung eines Entwurfs des Betreuungsgesetzes (BtG). Praxis Kinderpsychol Kinderpsychiatrie 1990;39:132-8.

Kunz J, et al. Zur Sterilisation geistig behinderter Patientinnen. Schweiz Med Wochenschr 1991;121:1328-35.

Goldhar J. The sterilization of women with an intellectual disability. Unversity of Tasmania Law Review 1991;10:157-96.

Ehrenström P. Stérilisation opératoire et maladie mentale. Une étude de cas. Gesnerus 1991;48:503-16.

Walter J (Hrsg.). Sexualität und geistige Behinderung. Heidelberg: HVA Edition Schindele; 1992.

Giami A, Lavigne C. La stérilisation des femmes handicapées mentales et le consentement libre et éclairé. Rev Méd Psychosom 1993;35:35-46.

Cepko R. Involuntary sterilization of mentally disabled women. Berkeley Women's Law Journal 1993;122-65.

Hiersche HD, Hiersche F. Die Sterilisation geistig Behinderter. Gynäkologie 1995;28:452-8.

CCNE Comité Consultatif National d'Ethique pour les Sciences de la Vie et de la Santé. Rapport: La contraception chez les personnes handicapées mentales. France, 1996.

Hofmann B. Sterilisation geistig behinderter Erwachsener. Baden-Baden: Nomos Verlag; 1996.

Gasser J, Heller G. Etude de cas: les débuts de la stérilisation légale des malades mentaux dans le canton de Vaud. Gesnerus 1997;54:242-50.

AEP Association Européenne de Psychiatrie. Symposium octobre 1997, Strasbourg. Giami A. Stérilisation et sexualité des personnes handicapées mentales.

AEP Association Européenne de Psychiatrie. Symposium octobre 1997, Strasbourg. Lachaux B, Renaud V. La contraception et la stérilisation des malades mentaux: au delà du manichéisme, quelques repères.

Ratzel R. Forensische Aspekte in der Gynäkologie - Sterilisation. GynSpectrum 1998;2:17-9. 